\title{
International Workshop on Future Internet and Smart Networks
}

\author{
Alexandre Santos, Pascal Lorenz and António Costa
}

\begin{abstract}
The International Workshop on Future Internet and Smart Networks, FI\&SN'2015, has been organized in conjunction by the Computer Communications and Networks Group, Centro ALGORITMI, University of Minho, Portugal and University of Haute Alsace, France.
\end{abstract}

Future Internet is a very meaningful name for the real evolution of Internet technology nowadays. Internet should no longer mean the concept of having a desktop or portable computer, or even any smart device, connected to plenty other of such systems, servers, or to the cloud.

Future Internet is to be the place where smart objects, smart phones, smart vehicles, computers, grids, clouds, cities, etc, are to be interconnected using dynamic and evolving intelligent network solutions. Underlying the possibility of having everything interconnected-computers, devices, things and people - and being able to ubiquitously exchange useful information, even in mobility, there should be highspeed broadband links to enable Smart Networks.

Smart Networks solutions here discussed range from Software Defined Networks to Named Data Networking and also from Network Resilience Optimization to Automated Network Management. As an important sum up, an insight into the challenges and key enabling technologies for a People-Centric Internet of Things, leading the way to a people-centric society in the near future, is presented and discussed.

\footnotetext{
A. Santos ( $)$ A. Costa

Centro ALGORITMI, University of Minho, Braga, Portugal

e-mail: alex@di.uminho.pt

A. Costa

e-mail: costa@di.uminho.pt

P. Lorenz

University of Haute Alsace, Mulhouse Cedex, France

e-mail: pascal.lorenz@uha.fr 